\title{
Análise Pré e Pós-Operatória da Capacidade Funcional e Qualidade de Vida de Pacientes Portadores de Osteoartrose de Quadril Submetidos à Artroplastia Total ${ }^{*}$
}

\section{Pre- and Post-Surgery Analysis of Functional Capacity and Quality of Life of Patienst with Osteoarthritis Submitted to Total Hip Artroplasty}

\author{
Lislei Jorge Patrizzi ${ }^{(1)}$, Karla Helena Coelho Vilaça ${ }^{(2)}$, Edmilson Takehiro Takata $^{(3)}$ e Gustavo Trigueiro ${ }^{(3)}$
}

\section{RESUMO}

Objetivo: avaliar a capacidade funcional da articulação do quadril e a qualidade de vida de pacientes portadores de osteoartrose submetidos à artroplastia total. Métodos: foram avaliados 12 pacientes através do questionário Harris Hip Score (modificado/ escala de 0-95 pontos) e goniometria no pré e pós-operatório de artroplastia total de quadril. Resultados: a amplitude de movimento mostrou-se incompleta em todos os arcos de movimento no pré-operatório. No pós-operatório os valores aumentaram significativamente na flexão $(p=0,007)$, extensão $(p=0,02)$, abdução $(p=0,001)$, rotação medial $(p=0,0002)$ e lateral $(p=0,01)$, apenas o aumento do movimento de adução de quadril não foi significativo $(p=0,08)$. A medida do escore obtido através do questionário no pré-operatório foi 39,7 pontos e no pósoperatório 74,1 pontos $(\mathrm{P}<0,000001)$. Conclusões: a artroplastia total do quadril proporciona ao paciente alívio da dor, melhora na realização das atividades da vida diária e aumento da amplitude do movimento articular do quadril. Os pacientes, porém, relatam medo e insegurança na realização dessas atividades.

Palavras-chave: osteoartrose, quadril, artroplastia, qualidade de vida, reabilitação, tratamento.

\section{INTRODUÇÃO}

A osteoartrose caracteriza-se patologicamente por degeneração focal da cartilagem articular, espessamento ósseo subcondral e proliferações osteocondrais marginais ${ }^{(1)}$.

\begin{abstract}
Objective: To evaluate the functional capacity of the hip articulation and the quality of life of patients with osteoarthritis submitted to total artroplasty. Methods: 12 patients were assessed through the questionnaire Harris Hip Score (modified/scale of 0-95 points) and goniometry in the pre- and post-surgery of total hip artroplasty. Results: The pre-surgery questionnaire score was 39.7 and in the postsurgery the average score reached 74.1 points $(p=0.000001)$. The range of motion of the hip was incomplete in all the movement arches in the pre-surgery, but in the post-surgery this value increased significantly in flexion $(p=0.007)$, extension $(p=0.02)$, abduction ( $p=0.001$, medial rotation $(p=0.0002)$ and lateral rotation $(p=0.01)$; only the aduction movement was not significantly increased $(p=0.08)$. Conclusions: Total hip artroplasty reliefs patients pain, improves daily life activities and increases the hip range of motion; patients, however, relate fear and insecurity in the accomplishment of those activities.
\end{abstract}

Keywords: Osteoarthritis, hip, artroplasty, quality of life, rehabilitation, treatment.

Apresenta-se clinicamente com episódios recidivantes de dor, sinovite com ou sem derrame, rigidez e limitação progressiva de movimentos ${ }^{(2,3,4)}$.

De acordo com Buckwalter e Martin ${ }^{(5)}$, o início de um programa de tratamento na fase inicial da doença articular

\footnotetext{
* Departamento de ortopedia e traumatologia e setor de fisiatria da Universidade Federal de São Paulo/Escola Paulista de Medicina (Unifesp/EPM). Recebido em 27/03/2003. Aprovado, após revisão, em 29/12/2003.

1. Docente do departamento de reumatologia da Universidade de Franca, SP.

2. Residente do departamento de fisioterapia geriátrica da Universidade de Franca, SP.

3. Departamento de ortopedia e traumatologia/setor quadril adulto da Unifesp/EPM, São Paulo, SP.

Endereço para correspondência: Lislei Jorge Patrizzi. Universidade de Franca - Unifran, R. Mauro Antônio Comparini 320, ap. 5, Franca, SP, CEP 14403-123. E-mail: lpatrizzi@uol.com.br
} 
pode causar uma diferença significativa na qualidade de vida do paciente, contribuindo para reduzir a progressão da enfermidade. $O$ tratamento deve seguir de acordo com a gravidade do acometimento e com a expectativa do paciente. Deve ser multidisciplinar, buscando a melhora funcional, mecânica e clínica. O tratamento não farmacológico envolve programas educativos, exercícios terapêuticos associados aos recursos eletrotermoterápicos, hidroterapia, uso de órteses e equipamentos (palmilhas, bengalas, etc.). O tratamento farmacológico envolve analgésicos e antiinflamatórios, agentes tópicos, drogas sintomáticas de ação duradoura, cloroquina e terapia intra-articular. Em pacientes acometidos com osteoartrose avançada, que já apresentam alguma deformidade física, perda da função, inabilidade para realizar as atividades diárias ou quando o tratamento conservador se mostra ineficaz, os tratamentos cirúrgicos que envolvem osteotomias, desbridamentos artroscópicos e artroplastias podem aliviar e melhorar a função articular do paciente ${ }^{(2,3,5,6)}$.

$\mathrm{Na}$ evolução das técnicas operatórias do aparelho locomotor, destacam-se as relacionadas com a substituição da articulação do quadril que, apesar de ser um procedimento radical, melhora a qualidade de vida dos pacientes, com retorno às atividades da vida diária e até mesmo às atividades laborativas ${ }^{(7,8,9)}$.

A justificativa do trabalho é quantificar o ganho funcional e conseqüente qualidade de vida em pacientes submetidos à artroplastia total do quadril.

\section{PACIENTES E MÉTODOS}

O estudo baseia-se na observação pré-operatória e pós-operatória (60. ${ }^{\circ}$ dia) de 12 pacientes portadores de osteoartrose do quadril, submetidos à artroplastia total primária.

Os critérios de inclusão e exclusão previamente estabelecidos estipulam que os pacientes devam ser portadores de osteoartrose idiopática ou secundária diagnosticada através de exame radiográfico (diminuição do espaço articular importante associado às áreas de esclerose e formação osteofitária) e clínico (dor intensa comprometendo funções e marcha), com indicação cirúrgica de artroplastia total do quadril e com um mínimo de 20 pontos no questionário sobre dor e atividades da vida diária (modificado - Harris Hip Score $)^{(10)}$.

A Tabela 1 apresenta os dados relativos aos pacientes avaliados com relação ao número de ordem, sexo, idade, profissão, diagnóstico e lado operado, sendo 6 (50\%) pacientes do sexo feminino e $6(50 \%)$ do sexo masculino,
TABela 1

DADOS RELATIVOS AOS 12 PACIENTES SUBMETIDOS À ARTROPLASTIA TOTAL DO QUADRIL POR VIA LATERAL, SEGUNDO O NÚMERO DE ORDEM, SEXO, IDADE, DIAGNÓSTICO E LADO OPERADO.

\begin{tabular}{ccccc}
\hline $\mathbf{N}$ & Sexo & Idade & Diagnóstico & Lado \\
\hline 1 & $\mathrm{~F}$ & 81 & OA & $\mathrm{E}$ \\
2 & $\mathrm{M}$ & 51 & $\mathrm{OA}$ & $\mathrm{D}$ \\
3 & $\mathrm{~F}$ & 68 & $\mathrm{OA}$ & $\mathrm{E}$ \\
\hline 4 & $\mathrm{~F}$ & 72 & $\mathrm{OA}$ & $\mathrm{D}$ \\
\hline 5 & $\mathrm{~F}$ & 88 & $\mathrm{OA}$ & $\mathrm{D}$ \\
\hline 6 & $\mathrm{M}$ & 75 & $\mathrm{OA}$ & $\mathrm{E}$ \\
7 & $\mathrm{M}$ & 41 & $\mathrm{OA}$ & $\mathrm{D}$ \\
\hline 8 & $\mathrm{~F}$ & 74 & $\mathrm{OA}$ & $\mathrm{D}$ \\
\hline 9 & $\mathrm{M}$ & 83 & $\mathrm{OA}$ & $\mathrm{D}$ \\
\hline 10 & $\mathrm{~F}$ & 68 & $0 \mathrm{~A}$ & $\mathrm{E}$ \\
11 & $\mathrm{M}$ & 64 & $0 \mathrm{~A}$ & $\mathrm{E}$ \\
\hline 12 & $\mathrm{M}$ & 64 & $0 \mathrm{~A}$ & $\mathrm{E}$ \\
\hline
\end{tabular}

(M): masculino; (F): feminino; (OA): osteoartrose; (E): esquerdo; (D): direito

com idades entre 41 e 88 anos, idade média de 69,08 anos e mediana de 73 anos. Quanto ao diagnóstico, 10 (83,3\%) pacientes apresentam osteoartrose idiopática e $2(16,7 \%)$ osteoartrose secundária à necrose asséptica da cabeça femoral, sendo $6(50 \%)$ do lado esquerdo e $6(50 \%)$ do lado direito.

\section{QUESTIONÁRIO SOBRE DOR E ATIVIDADES DA VIDA DIÁRIA (AVD) - HARRIS HIP SCORE (1969) MODIFICADO}

O Harris Hip Score consiste em uma escala de pontuação de 0-100, que avalia vários parâmetros como dor, função, amplitude de movimento articular e deformidades.

A amplitude de movimento do quadril foi efetuada separadamente do questionário, pois notamos a necessidade de uma avaliação mais completa desta articulação, dando ênfase aos aspectos funcionais dos movimentos. Em decorrência desta adaptação, a escala de pontuação do questionário de 0-100 foi modificada para 0-95 pontos (Tabela 2).

A dor recebe até 44 dos 95 pontos possíveis, sendo graduada em ausente, discreta, fraca, moderada, acentuada e incapacitante. A função é avaliada segundo as atividades da vida diária e a marcha.

O item atividades da vida diária recebe 14 pontos, dentro dos 47 possíveis para a avaliação da função, incluindo forma e necessidade de auxílio para subir escadas, uso de transporte 
Tabela 2

“Harris Hip Score” (modificado) (J Bone Joint Surg Am 51: 737-55, 1969.)

\begin{tabular}{|c|c|c|c|}
\hline \multicolumn{4}{|c|}{ DOR } \\
\hline \multicolumn{2}{|c|}{ Nenhuma } & \multicolumn{2}{|l|}{44} \\
\hline & Fraca ("sempre, mas fraca”) & \multicolumn{2}{|l|}{30} \\
\hline & Moderada ("sempre e incômoda, mas suportável”) & \multicolumn{2}{|l|}{20} \\
\hline \multicolumn{4}{|c|}{ FUNÇÃO } \\
\hline AVDs & \multicolumn{2}{|l|}{ Atividade } & Pontos \\
\hline Escada & \multicolumn{2}{|l|}{ Pé após pé sem corrimão } & 04 \\
\hline Sentar & Uma hora qualquer cadeira & & 05 \\
\hline & Meia hora cadeira alta & & 03 \\
\hline & Impossivel & & 00 \\
\hline Sapato (de amarrar) meias & Com facilidade & & 04 \\
\hline & Com dificuldade & & 02 \\
\hline & Incapaz & & 00 \\
\hline Marcha & Descrição & & Pontos \\
\hline Claudicação & Ausente & & 11 \\
\hline & Discreta & & 08 \\
\hline & 2 muletas & & 00 \\
\hline & Incapaz & & 00 \\
\hline & Especificar - & & \\
\hline Distância & Ilimitada & & 11 \\
\hline & 6 quarteirões & & 08 \\
\hline & 2 ou 3 quarteirões & & 05 \\
\hline & Somente dentro de casa & & 02 \\
\hline & Restrito cama e cadeira & & 00 \\
\hline & & Pontos & \\
\hline Ausent & & 04 & \\
\hline$+30^{\circ} \mathrm{d}$ & & 00 & \\
\hline$+10^{\circ} \mathrm{d}$ & & 00 & \\
\hline$+10^{\circ} \mathrm{d}$ & ensão & 00 & \\
\hline Discre & $3,2 \mathrm{~cm}$. & 00 & \\
\hline
\end{tabular}


público, permanência na posição sentada e possibilidade de calçar sapatos e meias. O item marcha recebe 33 pontos, dentro dos 47 estipulados para a avaliação da função. Os aspectos analisados incluem claudicação, podendo ser ausente, discreta, moderada e grave, e necessidade de suporte para a marcha, incluindo nenhum auxílio, uma bengala e distâncias variadas, uma muleta, duas muletas e incapacidade para a marcha. Na avaliação das alterações articulares, essa escala considera ausência de anquilose, flexão fixa de mais de $30^{\circ}$ no quadril, adução fixa de $10^{\circ}$ no quadril, rotação interna fixa de $10^{\circ}$ no quadril e discrepância de comprimento de membros maior que $3,2 \mathrm{~cm}$.

\section{GONIOMETRIA}

O goniômetro usado neste estudo é o chamado goniômetro universal. Tomamos como base os valores seguidos pela American Academy Orthopaedic Surgeons (1965), e The Veterans Administration of United States of North America $(1963)^{(11,12,13)}$.

Todos os pacientes foram avaliados quanto à amplitude de movimento articular (ADM) no pré-operatório e pósoperatório. Foram realizadas as medidas de amplitude de movimento de abdução do quadril (Tabela 3) com o paciente em decúbito dorsal.

A análise estatística foi realizada seguindo o teste-t de Student, sendo estatisticamente significativos para $\mathrm{p}<0,05$.

\section{RESULTADOS}

No pré-operatório, $8(66,7 \%)$ pacientes apresentaram dores acentuadas, e no pós-operatório, $10(83,3 \%)$ pacientes relataram ausência de dor. Quando comparados os dados, observa-se que apenas $1(8,3 \%)$ paciente era capaz de subir escadas no pré-operatório sem auxílio do corrimão e no pós-operatório $3(25 \%)$ pacientes conseguiram realizar essa atividade. Dos 7 (58,3\%) pacientes que no pré-operatório apresentavam dificuldade para subir escadas, apenas 4 $(33,3 \%)$ o faziam com a mesma dificuldade no pósoperatório. Observou-se a permanência de incapacidade para subir escadas no pós-operatório em $2(16,7 \%)$ pacientes, que relataram medo de cair ao realizar essa atividade. Quanto à utilização de transporte público no pósoperatório não foi notada muita diferença. Vale ressaltar, entretanto, que no período de 60 dias do pós-operatório, os pacientes foram orientados a ter cuidados e prudência. Assim, alguns pacientes, naquele período, apresentaramse com auxílio para a marcha e/ou acompanhante, e referiam evitar o uso de transporte público.
TABELA 3

VALORES EM GRAUS DOS MOVIMENTOS DA ARTICULAÇÃO DO QUADRIL NORMAL

\begin{tabular}{lcc}
\hline Articulação & Movimento & Graus de Movimento \\
\hline Quadril & Flexão & $0-125$ \\
& Extensão & $0-10$ \\
& Adução & $0-15$ \\
& Abdução & $0-45$ \\
& Rotação medial & $0-45$ \\
& Rotação lateral & $0-45$ \\
\hline
\end{tabular}

No pré-operatório $7(58,3 \%)$ pacientes conseguiam permanecer sentados por uma hora em qualquer cadeira, no pós-operatório este número elevou-se para 11 (91,7\%) pacientes. Amarrar os sapatos foi outra atividade avaliada no pré-operatório e comparada no pós-operatório. Enquanto apenas $1(8,3 \%)$ paciente realizava esta atividade no pré, $3(25 \%)$ pacientes eram capazes de fazê-la no pósoperatório. Quanto à incapacidade para realizar esta atividade, no pré-operatório $5(41,7 \%)$ pacientes mostraram-se incapazes de amarrar os sapatos e no pós $6(50 \%)$ pacientes apresentaram esta incapacidade. Quanto à claudicação, todos os pacientes apresentaram-se claudicando no préoperatório; destes, $4(33,3 \%)$ pacientes apresentavam claudicação discreta, $6(50 \%)$ moderada e $2(16,7 \%)$ pacientes claudicação grave. No pós-operatório, dois $(16,7 \%)$ pacientes não apresentaram claudicação, $5(41,7 \%)$ pacientes apresentaram-na moderada e $5(41,7 \%)$ apresentaram claudicação discreta. Quanto à utilização de auxílio (bengala, muletas, andador) para a marcha, observou-se tanto no pré como no pós-operatório a permanência de 9 (75\%) pacientes necessitando de auxílio para a marcha e $3(25 \%)$ não. Foi notado também um aumento do número de pacientes capazes de realizar marcha sem limite de distância. No pré-operatório, $1(8,3 \%)$ paciente conseguia andar sem dor e sem distância limitada; já no pós-operatório, o número de pacientes capazes de realizar esta atividade elevou-se para $4(33,3 \%)$. Foi possível observar também que no pré-operatório, $66,7 \%$ andavam somente em casa e no pós-operatório esse número reduziu-se para $41,7 \%$.

Os resultados do questionário mostram no pré-operatório uma média de 39,7 pontos, mediana de 35,00, um desvio padrão de 11,8 e um desvio padrão com relação à média de 3,4. No pós-operatório a média teve um valor igual a 
74,2 pontos, mediana de 74,00, um desvio padrão de 11,2 e um desvio padrão com relação à média de 3,2. Os valores encontrados com relação ao intervalo de confiança com 95\% de significância para a diferença entre pares foram 44,27 e $-24,73$.

Os escores do questionário sobre dor e atividades da vida diária foram significativamente melhores no pós-operatório $(p=0,000001$ - teste $t)$ (Figura 1).

Quanto à capacidade funcional da articulação, foi observado no pré-operatório uma ADM incompleta nos movimentos de flexão, extensão, adução, rotação medial e lateral do quadril em todos os pacientes, e no pós-operatório houve um ganho significativo na amplitude dos movimentos de flexão $(p=0,007)$, extensão $(p=0,02)$, abdução $(\mathrm{p}=0,001)$, rotação medial $(\mathrm{p}=0,0002)$ e lateral $(\mathrm{p}=0,01)$; apenas o movimento de adução de quadril não foi significativo $(p=0,08)$. Até o dia da reavaliação os pacientes foram orientados a ter cautela na realização desse movimento (Tabela 4).

\section{DISCUSSÃO}

Vários estudos vêm sendo realizados, ao longo dos anos, com o intuito de proporcionar uma melhor qualidade de vida para os portadores de osteoartrose do quadril, uma vez que se trata de uma moléstia crônica, produto final da degradação tissular cartilaginosa que causa dor, incapacidade articular e conseqüente comprometimento da qualidade de vida ${ }^{(14,15,16,17)}$.

De acordo com Jakobsson e Hallberg ${ }^{(18)}$, a dor articular e a rigidez características da osteoartrose, levam principalmente os pacientes idosos a uma intensa limitação funcional e restrição de movimentos, acometendo diretamente a qualidade de vida dessas pessoas.

O Harris Hip Score é amplamente utilizado na avaliação funcional de pacientes portadores de deficiências do quadril, tendo alta validade e confiabilidade em todos os itens de pontuação, principalmente de dor e função ${ }^{(19)}$; no entanto, há outros questionários que avaliam o bem-estar e a qualidade de vida e que podem ser utilizados ${ }^{(20,21)}$. Utilizou-se

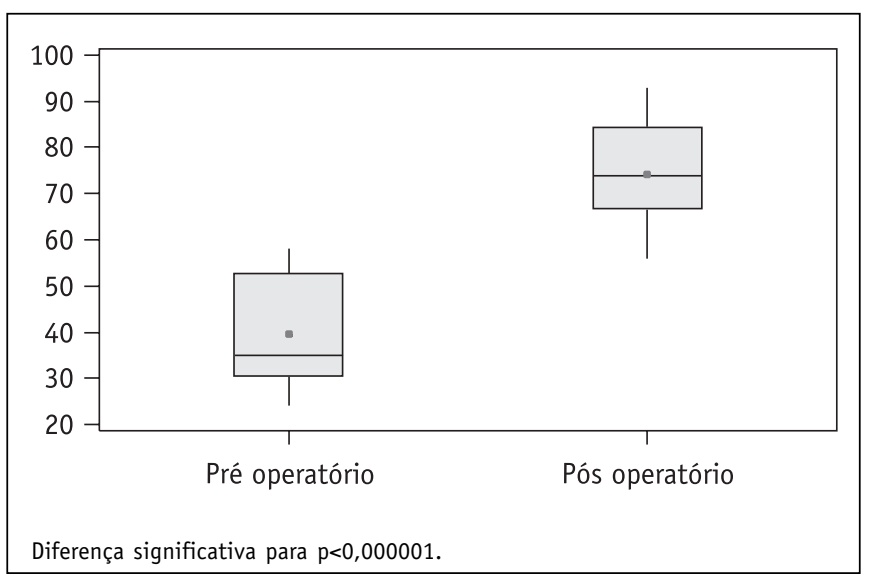

FiguRa 1- Pontuação obtida no questionário Harris Hip Score no pré e pós-operatório.

tratamentos conservadores (incluindo medidas gerais, medicamentos, aparelhos ortopédicos e fisioterapia) e cirúrgicos são utilizados $^{(3,16,22,23,24)}$. Segundo Cecin, Galati e Ribeiro ${ }^{(23)}$, o tratamento convencional da osteoartrose baseia-se em combater sintomas e postergar a sua evolução. No que diz respeito à melhora dos sintomas e da função, o tratamento fisioterapêutico exerce papel muito importante. Marques e Kondo ${ }^{(24)}$ realizaram uma revisão da literatura a fim de identificar o efeito da fisioterapia em pacientes com osteoartrose. Os autores concluem que a fisioterapia age beneficamente na reabilitação dos pacientes, auxiliandoos tanto no alívio dos sintomas quanto na execução das atividades da vida diária, além de melhorar a funcionalidade e contribuir para manter a qualidade de vida. Sabe-se, entretanto, que para se obter melhora significativa de todos os sinais e sintomas apresentados pelos pacientes com osteoartrose avançada, faz-se necessária a substituição da articulação lesada ${ }^{(3,16,23,24)}$.

Nesse sentido, o tratamento cirúrgico por meio da artroplastia total do quadril proporciona alívio da dor, ganho de amplitude de movimento e, conseqüentemente, melhora das funções nos quadros de degeneração articular $\operatorname{grave}^{(25,26,27,28,29,30,31)}$. Com a experiência crescente adquirida

TABELA 4

Resultados DA Amplitude DE MOVimento articular (ADM) ATIVA DO QUADRIL

\begin{tabular}{|c|c|c|c|c|c|c|}
\hline & Flexão & Extensão & Adução & Abdução & Rotação medial & Rotação lateral \\
\hline Pré-operatório & $58,17 \pm 14,97$ & $1,67 \pm 3,26$ & $10,42 \pm 3,34$ & $11,92 \pm 6,22$ & $11,67 \pm 4,92$ & $17,50 \pm 10,98$ \\
\hline Pós-operatório & $77,50 \pm 16,99 *$ & $5,00 \pm 3,02 *$ & $11,67 \pm 3,26$ & $25,00 \pm 8,12^{*}$ & $23,75 \pm 7,72^{*}$ & $25,42 \pm 9,16^{*}$ \\
\hline
\end{tabular}

Valores expressos como médias $\pm \mathrm{DP}$.

*Diferença significativa para $\mathrm{p}<0,05$. 
quanto a esta cirurgia, muitas técnicas foram aprimoradas para a adequada exposição do quadril, com a obtenção de melhores resultados funcionais.

Utilizou-se o mínimo de 20 pontos dessa escala como critério de inclusão dos pacientes no estudo, pois fazia-se necessário o mínimo de funcionalidade no pré-operatório para a realização dos exames clínico-funcionais.

A destruição da cartilagem articular que atinge o osso subcondral, e a diminuição do espaço articular, evidente nos exames radiológicos dos pacientes com osteoartrose, resulta em diminuição da $\mathrm{ADM}^{(22,23,24,32)}$. Essa diminuição dificulta as atividades funcionais, tal como avaliadas através do questionário escolhido para o presente estudo.

No pré-operatório, a dor causada por destruição articular dificulta atividades básicas e necessárias, como permanecer sentado por uma ou meia hora. Quanto à incapacidade para amarrar os sapatos, subir escadas e entrar em transporte público, os pacientes relatavam medo e dor ao sobrecarregar a articulação para a realização destas atividades. Quanto à claudicação, no pré-operatório, mais uma vez a dor e a destruição articular são fatores desencadeantes dessa condição. Gross, Fetto e Rosen ${ }^{(33)}$ afirmam que, sentindo dor no quadril (provocada pela osteoartrose), o paciente tenta reduzir a quantidade de tempo gasto no apoio sobre aquela região. $\mathrm{O}$ tronco é projetado lateralmente sobre o quadril durante a carga. Isto é feito na tentativa de reduzir a força compressiva dos músculos abdutores do quadril durante a carga. Os autores caracterizam esta alteração como marcha Trendelenburg compensada. Pode-se considerar que a ausência da dor, no pós-operatório, propicia marcha normal. Quanto à utilização de auxílio (bengala, muletas, andador) para a marcha, Gross, Fetto e Rosen ${ }^{(33)}$ ressaltam que uma bengala mantida na mão contralateral auxilia os músculos abdutores do quadril na resistência do movimento gravitacional, que puxa o corpo em direção ao lado não

\section{REFERÊNCIAS}

1. Chitnavis J, Sinsheimer JS, Suchard MA, Clipsham K, Carr AJ: Endstages coxartrhosis. Aetiology, clinical patterns and radiological features of idiopathic osteoarthritis. Rheumatol 39: 612-9, 2000.

2. Moreira C, Carvalho MAP: Noções Práticas de Reumatologia, Belo Horizonte, Editora Health, 1996.

3. Skare TL: Reumatologia/Princípios e Prática, Rio de Janeiro, Editora Guanabara Koogan, 1999.

4. Weinstein SL, Buckwalter JA: Ortopedia de Turek, 5.a ed, São Paulo, Editora Manole, 2000. sustentado durante a fase de oscilação. É válido ressaltar que todos os pacientes do presente estudo, mesmo após liberação médica para marcha sem apoio, relataram medo e insegurança. Observou-se também aumento no número de pacientes capazes de realizar marcha sem limite de distância.

Para todas as atividades da vida diária, faz-se necessária a amplitude de movimento o mais próximo da normalidade, facilitando funções como amarrar os sapatos, subir escadas, entrar em transporte público e permanecer na posição sentada, justificando a goniometria ser um dos procedimentos mais utilizados na avaliação como parte funcional da ciência fisioterapêutica ${ }^{(13)}$.

A atividade ocupacional dos pacientes foi questionada no pré-operatório com o objetivo de avaliar o retorno do paciente a uma vida normal ou próximo da normalidade. No 60.o dia de pós-operatório todos os pacientes relataram não haver retornado às suas atividades normais, mas mostraram-se dispostos para o reinício.

Tellinii ${ }^{(34)}$ e Coimbra et al. ${ }^{(35)}$ esclarecem que a ação fisioterapêutica no pré-operatório e no pós-operatório é de extrema importância, pois proporciona vantagens ao paciente tanto em termos da qualidade da recuperação como no menor tempo para obtê-la.

Freburger ${ }^{(36)}$ analisou a relação entre a utilização dos serviços fisioterapêuticos e os resultados dos pacientes após artroplastia total do quadril, concluindo que a intervenção fisioterapêutica é de extrema importância no processo de reabilitação geral do paciente.

Conclui-se que a artroplastia total do quadril devolve aos pacientes portadores de osteoartrose uma amplitude de movimento articular próxima da normalidade, aliviando a dor e facilitando a realização das atividades da vida diária. No entanto, esses pacientes referiram medo e insegurança na realização dessas atividades.

5. Buckwalter AJ, Martin J: Doença Articular Degenerativa. Clinical Symposia 47: 4-38, 1999.

6. Hawker GA, Wright JG, Coyte PC, et al: Determining the need for hip and knee arthroplasty: the role of clinical severity and patients' preferences. Medical Care 39: 206-16, 2001.

7. Lombardi JB, Laredo FJ, Wever HH, Molla CF, Lima JG: Artroplastia total do quadril não cimentada com a prótese de Roy Camille. F Med 95: 249-55, 1987.

8. Zavadak KH, Gibson KR, Wititley DM, Britz P, Kwoh, CK: Variability in the attainment of functional milestones during the care admission after total joint replacement. J Rheumatol 22: 482-7, 1995. 
9. Trigueiro G: Estudo clínico e radiográfico do fêmur nas artroplastias totais do quadril com a haste não cimentada de Spotorno, São Paulo, 1997, 116p, (Tese de Doutorado - Universidade Federal de São Paulo - Escola Paulista de Medicina).

10. Harris WH: Traumatic arthritis of the hip after deslocation and acetabular fractures: treatment by mold arthroplasty. J Bone Joint Surg 51: 737-55, 1969.

11. Lacôte M, Chevalier AM, Miranda A, Bleton J, Stevenin P: Avaliação Clínica da Função Muscular, São Paulo, Editora Manole, 1987.

12. Hoppenfeld S: Propedêutica - Coluna e Extremidades, 5.a ed, São Paulo, Livraria Atheneu, 1994.

13. Marques AP, Kondo A: Manual de Goniometria, São Paulo, Editora Manole, 1997.

14. Belhorn LR, Hess EV: An update on osteoarthritis. Rev Bras Reumatol 33: 181-6, 1993.

15. Vellutini WC: Envelhecimento e degeneração da cartilagem articular. Acta Ortop Bras 5: 43-6, 1997.

16. Fernandez RN, Ximenes AC, Rassi CM, Silva NA: Perspectivas terapêuticas para a osteoartrose. Rev Bras Reumatol 37: 97-102, 1997.

17. Rezende MU, Hernandez AJ, Camanho GL, Amatuzzi MM: Cartilagem articular e osteoartrose. Acta Ortop Bras 8:100-4, 2000.

18. Jakobsson ULF, Hallberg I: Pain and quality of life among older with rheumatoid arthritis and/or osteoarthritis: a literature review. Journal of Clinical Nursing 11: 430-43, 2002.

19. Soderman P, Malchau H: Is the Harris hip score system useful the outcome of total hip replacement? Clinical Orthopaedics and Related Research 384: 189-97, 2001.

20. Lerner D, Reed JI, Massarotti E, Wester LM, Burke TA: The work limitations questionnaire's validity and reliability among patients with osteoarthritis. J Clin Epidemiology 55: 197-208, 2002.

21. Groessl EJ, Kaplan R, Cronan TA: Quality of well-being in older people with osteoarthritis. Arthritis Rheum 49: 23-8, 2003.

22. Greve JMD, Plapler PG, Seguchi HH, Pastor EH, Baptisttella LR: Tratamento fisiátrico da dor na osteoartrite. Rev Hosp Clín Fac Med S Paulo 47: 185-9, 1992.
23. Cecin HA, Galati MC, Ribeiro ALP, Cecin AO: Reflexões sobre a eficácia do tratamento fisiátrico da osteoartrose. Rev Bras Reumatol 35: 270-8, 1995.

24. Marques AP, Kondo A: A fisioterapia na osteoartrose: uma revisão da literatura. Rev Bras Reumatol 38: 83-90, 1998.

25. Johnston RC: Clinical follow-up of total hip replacement. Clin Orthop 95: 118-26, 1973.

26. Visuri T, Honkanen R: The influence of total hip replacement on selected activities of daily living and on the use of domestic aid. Scand J Rehab Med 10: 221-5, 1978.

27. Wilcock GK: Benefits of total hip replacement to older patients and the communit. BMJ 2: 37-9, 1978.

28. Haworth RJ, Hopkins J, Ells P, Ackroyd CE, Mowat G: Expectations and outcome of total hip replacement. Rheumatol Rehabil 20: 65-70, 1981.

29. Wachelke AG, Matschinske CR: Fisioterapia no pós-operatório de artroplastia de quadril. Fisioter Mov 3: 81-7, 1990.

30. Pettine KA, Aamlid BS, Cabanela ME: Elective total hip arthroplasty in patients older than 80 years of age. Clin Orthop 266: 127-32, 1991.

31. Skinner HB: Patokinesiology and total joint arthroplasty. Clin Orthop Rel Res 288: 78-86, 1993.

32. Carvalho FET, Papaléo NM: Geriatria/Fundamentos, Clínica e Terapêutica. São Paulo, Editora Atheneu, 2000.

33. Gross J, Fetto J, Rosen E: Exame Musculoesquelético. Porto Alegre, Editora Artmed, 2000.

34. Tellini GG: Estudo crítico dos efeitos da cinesioterapia nos resultados funcionais obtidos em pacientes submetidos à prótese total do quadril por artropatias crônicas diversas. São Paulo, 1996, 116p (Tese de mestrado - Universidade Federal de São Paulo - Escola Paulista de Medicina).

35. Coimbra IB, Pastor EH, Greve JM, et al: Consenso brasileiro para o tratamento da osteoartrite (artrose). Rev Bras Reumatol 42: 371-4, 2002.

36. Freburger JK: An analysis of the relationship between the utilization of physical therapy services and outcomes of care for patients after total hip arthroplasty. Phys Ther 80: 448-58, 2000. 